\title{
Estudo de áreas e depósitos preferenciais de Aedes albopictus (Skuse, 1894) e Aedes aegypti (Linnaeus, 1762) no Município de Paracambi - Rio de Janeiro, Brasil
}

Vanessa Aparecida Ribeiro Canela Soares ${ }^{1}$, William Costa Rodrigues² \& Marise Maleck de Oliveira Cabral $^{3}$

1. Centro de Ciências Exatas, Tecnológicas e da Natureza e Unidade de Apoio ao Ensino, Universidade Severino Sombra, Av. Exp. Oswaldo de Almeida Ramos, 28o, Vassouras, RJ. e-mail: vanessacanela@bol.com.br 2. Universidade Severino Sombra e Instituto Superior de Tecnologia, Paracambi/ Fundação de Apoio à Escola Técnica, RJ, e-mail: wcrodrigues@ebras.bio.br. 3. Centro de Ciências Exatas, Tecnológicas e da Natureza e Unidade de Apoio ao Ensino, Pesquisa e Extensão Prof. Antonio O. Izolani, Universidade Severino Sombra, , Av. Exp. Oswaldo de Almeida Ramos, 280, Vassouras, RJ, e-mail: mmaleck@oi.com.br. Autor para correspondência

EntomoBrasilis 1(3): 63-68 (2008)

Resumo. A presença de Aedes albopictus (S.) e Aedes aegypti (L.) em área urbana representa um risco potencial do interrelacionamento dessa espécie de mosquito com a população. Este estudo teve como objetivo avaliar a presença do Ae. albopictus e de Ae. aegypti no município de Paracambi, Estado do Rio de Janeiro, Brasil. As amostras foram obtidas através de coletas das formas imaturas em depósitos e/ou criadouros (caixas d’água, cisternas, tanques, ocos de árvores, lixo, pneus, etc) em três áreas do município (urbana, rural e área de transição) no período de 12 meses. Os resultados mostraram que Ae. albopictus distribuiu-se igualmente nas áreas urbanas e rurais. A área urbana e a área de transição apresentaram uma maior positividade para Ae. aegypti. Os produtos de descartes, caracterizados por depósito de letra I = outros (artificiais especiais), foram os preferenciais para ambas as espécies de mosquito.

Palavras-Chave: Aedes aegypti, Aedes albopictus, depósitos preferenciais, insetos vetores, desenvolvimento de larvas.

\section{Study of the preferred areas and deposits of Aedes albopictus (Skuse, 1894) and Aedes aegypti (Linnaeus, 1762) in the Paracambi city, Rio de Janeiro, Brazil.}

Abstract. The presence of Aedes albopictus (S.) and Aedes aegypti (L.) in urban areas represents a potential risk of inter-relationship of this species of mosquito with the population. This study aimed to evaluate the presence of Ae. albopictus and Ae. aegypti in the Paracambi city, Rio de Janeiro State, Brazil. Samples were obtained from collections of immature forms in deposits (water tanks, cisterns, tanks, hollow trees, trash, tires, etc.) in three areas of the city (urban, rural and the transition area) in the last 12 months. The results showed that Ae. albopictus is also distributed in urban and rural areas. The urban area and the area of transition had a higher positive for Ae. aegypti. They refused material that is the distinguished mark of letter I = other (artificial special) deposit has been preferred for both species of mosquito.

Key words: Aedes aegypti, Aedes albopictus, preferred deposits, vectors insects, larvae development.

$A$ edes aegypti Linnaeus trajetória evolutiva um 1762, desenvolveu em sua comportamento estritamente sinantrópico e antropofílico, sendo reconhecido com o hábito predominantemente urbano (Consoli \& LouRENÇo-DEOliveira 1994). Suas larvas podem ser facilmente encontradas em diversos tipos de criadouros naturais e/ou artificiais no domicílio e/ou peridomicílio (Focks et al. 1981; Lopes et al. 1993; Chiaravalloti Neto 1997, 2002; Souza-Santos 1999). A adaptação aos criadouros artificiais teria sido um grande passo em direção ao comportamento sinantrópico. Na atualidade, esse mosquito é altamente dependente dos recipientes manufaturados pelo homem (NATAL 2002). Picam na maioria das vezes no intradomicílio, em vôos próximos ao solo, em ambientes com pouca luz, e "vestidos" com uma camuflagem, representada pelas listras e manchas claras em fundo escuro. De alguma forma, supõem-se uma espécie que compartilhe o mesmo ambiente e os mesmos horários de atividades com o homem, tenha sido vantajoso no sucesso em obter sangue como alimento (NATAL 2002).

Aedes albobiptus Skuse, 1894, como vetor de arbovírus autóctones, ainda é uma incógnita. Todavia, a presença do Ae. albopictus nos diferentes pontos da mata, bem como na área antrópica, já capturados no interior de uma residência, denota um processo progressivo de colonização e evidencia a dispersão desse mosquito do ambiente silvestre para o urbano (Albuquerque et al. 2000). Embora no Brasil o Ae. albopictus não seja considerado transmissor de vírus do dengue, estudos de laboratório demonstraram a capacidade vetorial da população aos quatro sorotipos virais (MILLER \& BALLINGER 1988, Gomes et al. 1999). Este mosquito possui hábito diurno e sua distribuição no Brasil também está associada à presença do homem, utilizando como Ae. aegypti os criadouros gerados pela atividade humana e/ou criando-se em recipientes naturais e artificiais (Consoli \& OliveIRA 1994). Essa amplitude de distribuição e capacidade de adaptação a diferentes ambientes e situações determina dificuldades para o controle através da mesma metodologia utilizada para o Ae. aegypti.

O objetivo deste estudo foi avaliar a preferência pelo tipo de depósito, e as principais áreas de ocorrência do $A e$. aegypti e Ae. albopictus no município de Paracambi, Estado do Rio de Janeiro.

\section{Material e Métodos}

Caracterização do local de estudo. A área de estudo tem início no centro da cidade (área urbana) de Paracambi (Figuras 1 e 2) no Estado do Rio de Janeiro, Brasil, cortado pelo rio Sabuginho, e terminando em uma área envolvendo criação de animais, pastos, partes alagadas e morros intercalados com fragmentos de florestas, e caracterizada neste estudo como área rural. Esta área foi gradativamente sendo colonizada e conhecida como fazenda do Sabugo (Figura 2).

Levantamento. O levantamento foi realizado no ano de 2004, por um período consecutivo de doze meses. O bairro do Sabugo, 

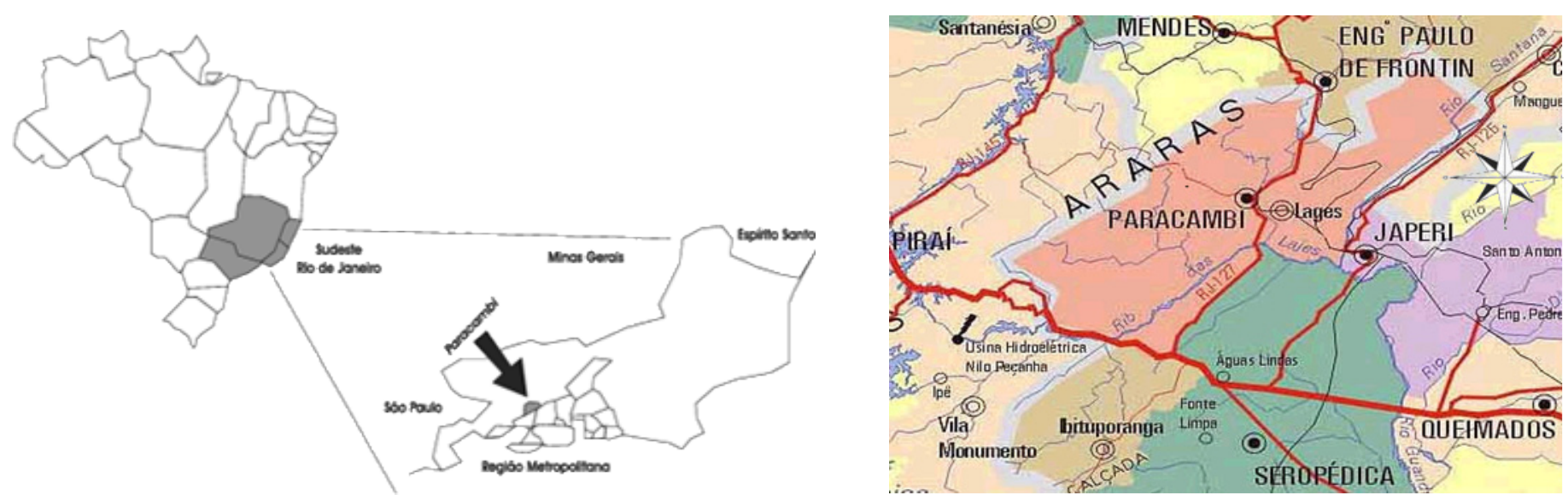

Figura 1. Localização geográfica do município de Paracambi, no Estado do Rio de Janeiro, Brasil.

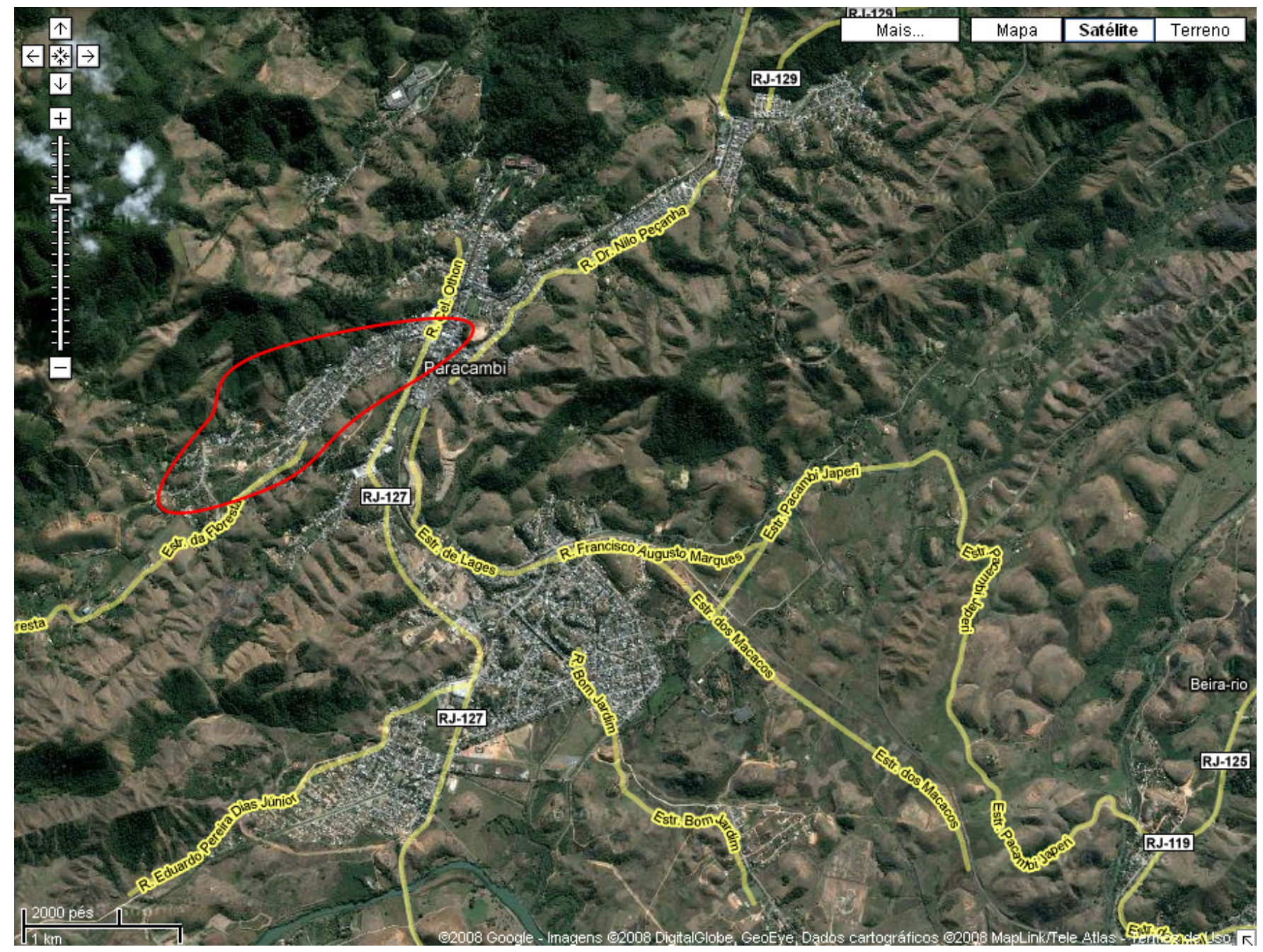

Figura 2. Localização da área de estudo, Bairro do Sabugo (destacado em vermelho), no município de Paracambi, no estado do Rio de Janeiro, Brasil. (Fonte: Google Maps (http://maps.google.com/maps?ll=-22.621555, $-43.710847 \& \mathrm{z}=12 \& \mathrm{t}=\mathrm{h} \& \mathrm{hl}=\mathrm{pt}-\mathrm{BR}$ )

área de estudo, está localizado no município de Paracambi, Estado do Rio de Janeiro, com 179,37 km² e 44.629 habitantes (IBGE, 2008). O bairro de estudo possui 8.054 imóveis distribuídos entre residências, terrenos baldios (TB), outros (igrejas, escolas, centro de religião afro-brasileira, fábricas, confecções de bolsas e roupas), e lojas comerciais. As coletas foram realizadas na área urbana, com treze quarteirões (1-13); área rural com sete quarteirões (14-20), e na área de interface entre a área urbana e rural, representado por apenas um quarteirão (quarteirão 42), situado no bairro de Guarajuba.

Utilizou-se para esse estudo o levantamento de pesquisa de focos larvários que tem como finalidade precípua conhecer a infestação e dispersão dos vetores e a detecção de depósitos para o Ae. aegypti e Ae. albopictus (TAveira et al. 2001, Donalísio \& GLASSER 2002). Os depósitos foram caracterizados como: (A) pneu; (B) tambor, tanque, barril, tina, tonel, depósito de barro; (C) vaso de planta; (D) material de construção, peças de carro; (E) garrafas, latas, plásticos; (F) poço, cisterna, cacimba; (G) caixa d'água; $(\mathrm{H})$ recipiente natural; ( $\mathrm{I}=$ outros) artificiais especiais; (J) armadilha; (K) "pool”. Os depósitos artificiais especiais, denominado neste levantamento como depósito I (outros), constara de sapatos velhos, ralos, bueiros, tampinhas de garrafas, casca de coco, freezer, partes de geladeiras, calhas de água, vasos sanitários sem uso, poças d' água, sapatas de obra, folhas de bananeira caídas no chão, casca de ovo e telhas.

Coleta. A identificação dos espécimes foi realizada pela observação direta dos caracteres morfológicos evidenciáveis ao microscópio estereoscópico, e ao microscópio de luz transmitida, utilizando a chave dicotômica proposta por ForATTINI (2002). Dentre os espécimes coletados, apenas os culicideos Ae. aegypti e o Ae. albopictus foram notificados. 
Análise dos resultados. Os resultados foram analisados quanto aos três tipos de ambientes: área urbana, área rural e zona de interface, e comparadas quanto a positividade de Ae. aegypti e Ae. albopictus por localidade (denominada como tratamento) e por tipo de depósitos (denominado de repetição). Os dados apresentaram distribuição não normal e foram analisados por variância não paramétrica, através de ANOVA de Friedman.

\section{Resultados}

A análise comparativa da presença de Ae. albopictus nas três diferentes localidades (área rural, área urbana e de interface) foi utilizada em três tratamentos, onze repetições, e dois como grau de liberdade. Os dados mostraram uma diferença significativa a $5 \%(\alpha=0,05)$ entre os locais, para a preferência do mosquito Ae. albopictus (Figura 1). Esse mosquito se adapta tanto na zona urbana $(\mathrm{x}=6,90 \pm 10,27)$ como na rural $(\mathrm{x}=5,81 \pm$ $10,42)$, ocorrendo em menor quantidade na zona de interface $(\mathrm{x}=$ $0,36 \pm 1,20$ ) (Figura 3).

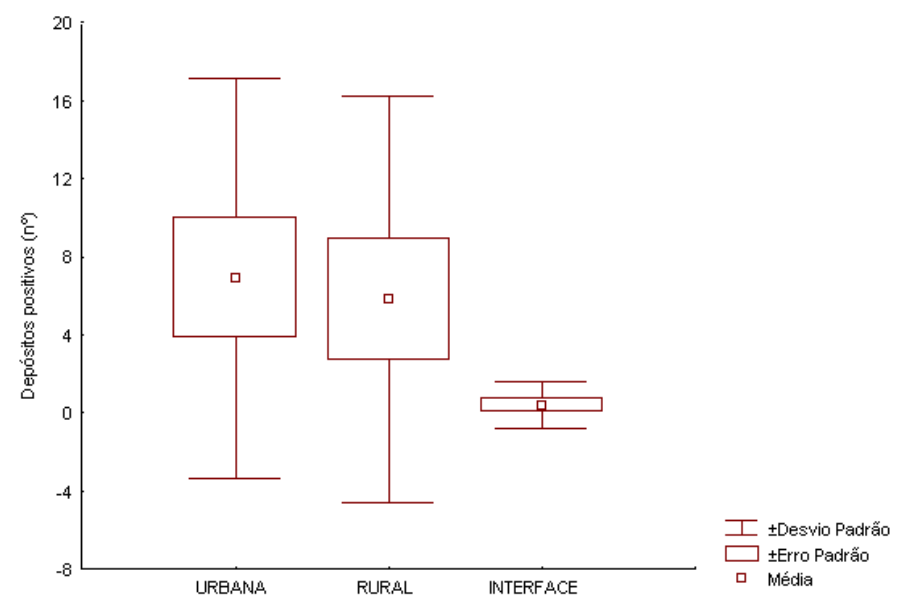

Figura 3. Incidência de Ae. albopictus no ano de 2004, nas três diferentes áreas (urbana, rural e interface), no bairro do Sabugo, município de Paracambi, RJ.

Entretanto, há uma distribuição equilibrada entre as áreas urbana e rural em relação à presença desse mosquito (Figura 3). Mesmo mostrando uma média maior para área urbana, quando comparada com a área rural, não há diferença na soma dos Ranks para o teste de Friedman. A fim de verificar a relação da ocorrência de Ae. albopictus entre as três áreas de estudo utilizou-se a análise de Cluster, e observou-se uma maior aproximação nas duas primeiras áreas, e com uma ligação mais restrita quando comparada com essa terceira área (Figura 4). Desta forma, o Ae. albopictus está presente tanto na área urbana como na rural, ou seja, não há preferência entre uma ou outra área de estudo. Com relação à positividade de Ae. albopictus houve uma preferência pelos depósitos artificiais especiais (depósito I = outros), tanto para a área urbana (38\%) quanto à área rural (55\%) (Tabela 1). Esse mosquito mostrou-se ainda presente em $53 \%$ dos diferentes depósitos na zona urbana, seguido de $44 \%$ desses depósitos da zona rural, por último a zona de interface não foi representativa para a positividade em comparação as áreas citadas (Tabela 1).
A análise comparativa da incidência de Ae. aegypti nas três diferentes localidades mostrou diferença significativa a $5 \%$ $(\alpha=0,05)$ entre os locais de preferência do Ae. aegypti. Os dados confirmaram a maior incidência desta espécie na área urbana $(\mathrm{x}=13,45 \pm 16,69)$, seguida pela área rural $(\mathrm{x}=5,45 \pm 8,27)$, e em menor número na zona de interface $(x=3,36 \pm 6,69)$ (Figura 5). A área urbana destacou-se das demais devido a uma maior variação dos dados quando comparada com as demais áreas.

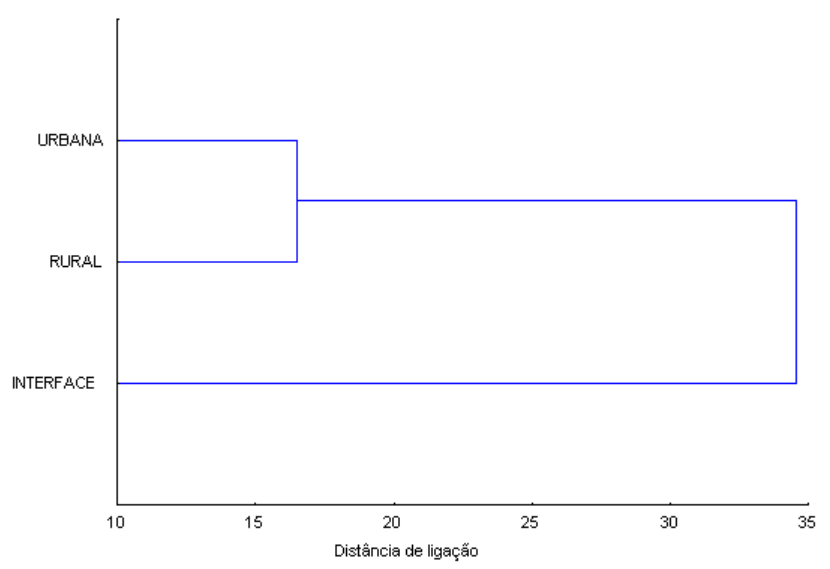

Figura 4. Áreas preferenciais de Ae. albopictus no bairro do Sabugo, Município de Paracambi, RJ, durante 2004.

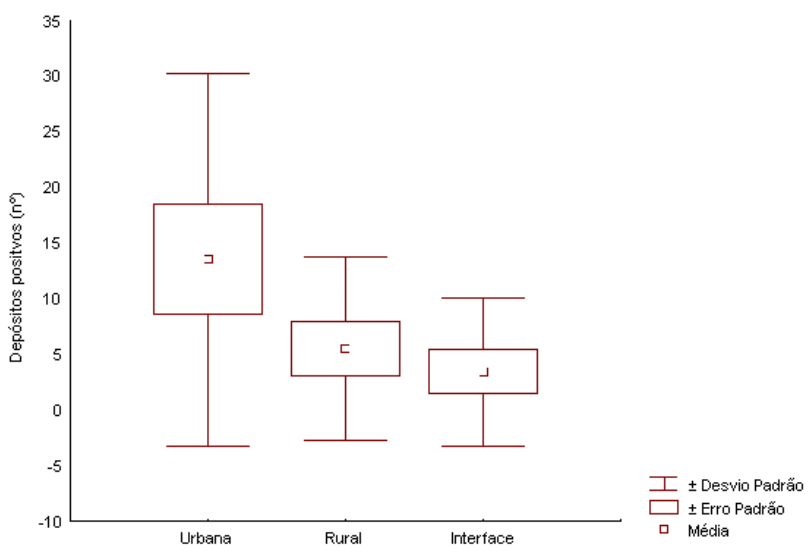

Figura 5. Incidência de Ae. aegypti no ano de 2004, nas três diferentes áreas (urbana, rural e interface), no município de Paracambi, RJ.

A análise de Cluster mostrou que a área rural e a área de interface encontram-se mais próximas e com uma ligação mais restrita quando comparada com a área urbana prevalecendo à preferência de Ae. aegypti pela área urbana (Figura 6). Quanto ao tipo de depósito houve uma preferência de Ae. aegypti também pelo tipo de depósito I de 34\%, 43\% e 56\% na área urbana, rural e de interface, respectivamente (Tabela 2), seguidos pela positividade em $22-23 \%$ no depósito $\mathrm{C}$ (vasos de plantas) tanto na área urbana quanto na área rural. Estes dados perfazem um total de 60\% de áreas positivas de Ae. aegypti na área urbana de acordo com os depósitos analisados.

A análise de variância de Friedman mostrou uma incidência maior deAe. aegypti $(\mathrm{x}=13,45 \pm 16,69)$ em comparação com Ae. albopictus $(\mathrm{x}=6,90 \pm 10,27)$ na área urbana (Tabela 3) $\mathrm{e}$

Tabela 1: Tipos e números de depósitos para a Ae. albopictus durante o ano de 2004, no bairro do Sabugo, Paracambi, RJ.

\begin{tabular}{|c|c|c|c|c|c|c|c|c|c|c|c|c|c|c|}
\hline \multirow{2}{*}{ Áreas } & \multirow{2}{*}{$\begin{array}{c}\text { Quarteirões } \\
\text { No }^{\circ}\end{array}$} & \multirow{2}{*}{$\begin{array}{c}\text { Imp } \\
N^{\circ} \\
\end{array}$} & \multicolumn{12}{|c|}{ Tipos de Depósitos (No) } \\
\hline & & & $\mathbf{A}$ & B & $\mathbf{C}$ & $\mathbf{D}$ & $\mathbf{E}$ & $\mathbf{F}$ & $\mathbf{G}$ & $\mathbf{H}$ & $\mathbf{I}$ & $\mathbf{J}$ & $\mathbf{K}$ & $\boldsymbol{\Sigma}$ \\
\hline Urbana & 11 & 51 & 1 & 9 & 16 & o & 20 & o & 1 & o & 29 & o & o & 76 \\
\hline Rural & 6 & 20 & 4 & 9 & 4 & 1 & 11 & o & o & 0 & 35 & 0 & 0 & 64 \\
\hline Interface & 1 & 35 & o & o & o & $\mathrm{o}$ & o & $\mathrm{o}$ & $\mathrm{o}$ & $\mathrm{o}$ & 4 & $\mathrm{o}$ & $\mathrm{o}$ & 4 \\
\hline$\Sigma$ & 18 & 106 & 5 & 18 & 20 & 1 & 31 & $\mathbf{o}$ & 1 & $\mathbf{o}$ & 68 & $\mathbf{0}$ & $\mathbf{0}$ & 144 \\
\hline
\end{tabular}

Legendas: (A) pneu; (B) tambor, tanque, barril, tina, tonel, depósitos de barro; (C) vaso de Planta; (D) material de construção, peças de carro; (E) garrafas, latas, plásticos; (F) poço, cisterna, cacimba; $(\mathrm{G})$ caixas d água; $(\mathrm{H})$ recipiente natural; (I= outros) artificiais especiais; (J) armadilha; (K) pool. Imp= imóveis positivos. 
Tabela 2. Tipos e números de depósitos positivos para a Ae. aegypti durante o ano de 2004, no Bairro de Sabugo, município de Paracambi, RJ.

\begin{tabular}{|c|c|c|c|c|c|c|c|c|c|c|c|c|c|c|}
\hline \multirow{2}{*}{ Áreas } & \multirow{2}{*}{$\begin{array}{c}\text { Quarteirões } \\
\text { No }^{\circ}\end{array}$} & \multirow{2}{*}{$\frac{\operatorname{Imp}}{\mathrm{N}^{\mathbf{0}}}$} & \multicolumn{12}{|c|}{ Tipos de Depósitos (No) } \\
\hline & & & $\mathbf{A}$ & B & $\mathbf{C}$ & $\mathbf{D}$ & $\mathbf{E}$ & $\mathbf{F}$ & G & $\mathbf{H}$ & $\mathbf{I}$ & $\mathbf{J}$ & $\mathbf{K}$ & $\Sigma$ \\
\hline Urbana & 12 & 112 & 2 & 25 & 33 & 3 & 20 & 4 & 4 & 2 & 51 & 4 & o & 148 \\
\hline Rural & 4 & 53 & 3 & 11 & 14 & 2 & 2 & 1 & 1 & o & 26 & o & o & 60 \\
\hline Interface & 1 & 38 & o & 11 & 1 & o & o & $\mathrm{o}$ & 3 & 1 & 21 & o & o & 37 \\
\hline$\Sigma$ & 17 & 203 & 5 & 47 & 48 & 5 & 22 & 5 & 8 & 3 & 98 & 4 & $\mathbf{0}$ & 245 \\
\hline
\end{tabular}

Legendas: (A) pneu; (B) tambor, tanque, barril, tina, tonel, depósitos de barro; (C) vaso de Planta; (D) material de construção, peças de carro; (E) garrafas, latas, plásticos; (F) poço, cisterna, cacimba; $(\mathrm{G})$ caixas d água; (H) recipiente natural; (I= outros) artificiais especiais; $(\mathrm{J})$ armadilha; (K) pool. Imp= imóveis positivos.

não houve diferença na incidência entre os Ae. aegypti $(\mathrm{x}=5,45$ $\pm 8,27)$ e Ae. albopictus $(\mathrm{x}=5,81 \pm 10,42)$ na área rural (Tabela 3). $\mathrm{Na}$ área de interface houve uma positividade maior para $A e$. aegypti $(\mathrm{x}=3,36 \pm 6,69)$ quando comparado a Ae. albopictus $(\mathrm{x}$ $=0,36 \pm 1,20)$ (Tabela 3).

Tabela 3. Média de incidência de Ae. aegypti e Ae. albopictus nas áreas urbana, rural e de interface, no bairro do Sabugo, município de Paracambi, RJ, no período de 2004.

\begin{tabular}{lccc}
\hline \multicolumn{1}{c}{ Espécie } & $\mathrm{x}$ & $\Sigma$ dos Rank's & $\mathrm{x} \pm \mathrm{DP}$ \\
\hline & Área urbana & Área urbana & Área urbana \\
\hline Ae. aegypti & 1,91 & 21,00 & $13,45 \pm 16,69$ \\
Ae. albopictus & 1,09 & 12,00 & $6,90 \pm 10,27$ \\
$\chi^{2}$ & 3,84 & $\chi^{2}$ Calculado & $9,0^{* *}$ \\
\hline & Área Rural & Área Rural & Área Rural \\
\hline Ae. aegypti & 1,59 & 17,50 & $5,45 \pm 8,27$ \\
Ae. albopictus & 1,40 & 15,50 & $5,81 \pm 10,42$ \\
$\chi^{2}$ & 3,84 & $\chi^{2}$ Calculado & $0,50 n s$ \\
\hline & Área de & Área de & Área de \\
& Interface & Interface & Interface \\
\hline Ae. aegypti & 1,72 & 19,00 & $3,36 \pm 6,69$ \\
Ae.albopictus & 1,27 & 14,00 & $0,36 \pm 1,20$ \\
$\chi^{2}$ & 3,84 & $\chi^{2}$ Calculado & $5^{*}$ \\
\hline
\end{tabular}

*Significativo a $5 \%(\alpha=0,05)$ pela tabela de valores críticos de $\chi^{2}$. ANOVA $(\mathrm{N}=11, \mathrm{df}=1)$.

**Significativo a $1 \%(\alpha=0,05)$ pela tabela de valores críticos de $\chi^{2}$. ANOVA $(\mathrm{N}=11, \mathrm{df}=1)$.

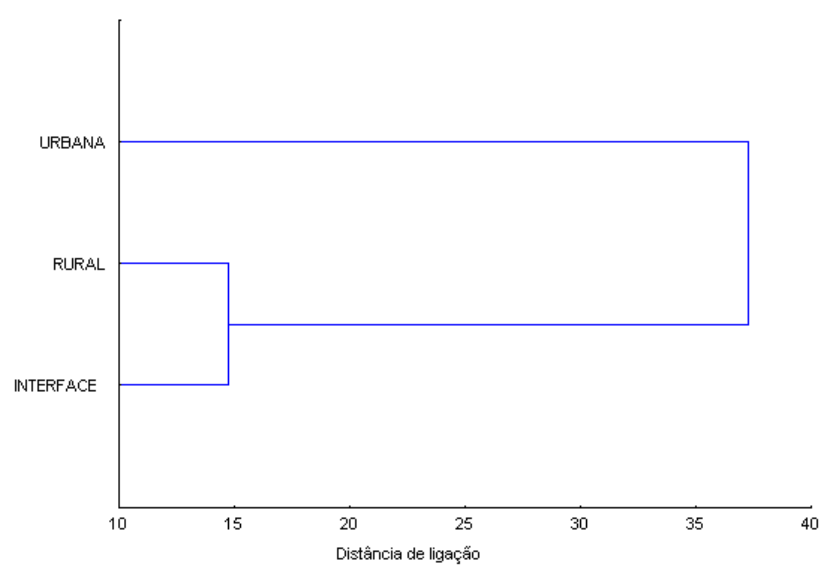

Figura 6. Áreas preferenciais de Ae. aegypti no bairro do Sabugo, Município de Paracambi, RJ, durante 2004.

\section{Discussão}

Com o advento da revolução industrial, a sociedade industrial - urbana teve seus costumes modificados, surgindo uma população altamente consumista, e dessa maneira, o homem passou a produzir grande quantidade de embalagens e recipientes descartáveis entre ferro, plástico, borracha, vidro, alumínio, latas e outros materiais. Tais artefatos são disponibilizados no ambiente gerando condições favoráveis à proliferação de mosquitos. Alguns autores evidenciam a inter-relação entre a urbanização e a domiciliação dos insetos que se adaptaram ao ambiente urbano, tornando-se pragas, ou vetores de doenças, constituindo problemas de Saúde Publica em vários países. Em relação aos mosquitos destaca-se o processo sinantrópico resultando eventos epidêmicos de grande magnitude para a Saúde Pública (Frankie \& Ehler 1978; Knudsen \& Slooff 1992).

As formas imaturas de Ae. albopictus possuem como microhabitats naturais os ocos de árvores e entrenós de bambus, além de uma grande diversidade de recipientes artificiais (HAWLEY 1988; LoPEs 1993). No Brasil, como espécie fitotelmata foi encontrada em ocos de árvores e internódios de bambu (Gomes et al. 1992; Gomes \& Marques 1988), bromélias (Ferreira Neto et al. 1987; GonÇalves \& Messias 2008; Forattini et al. 1998) e registrada colonizando bambus e pneus em um remanescente da Mata Atlântica da área urbana de Recife, estado de Pernambuco (Albuquerque et al. 2000). Gilotra et al. (1967) e O'Meara et al. (1995), apontaram que na zona urbana, o Ae. albopictus utiliza como criadouro os mesmos depósitos artificiais utilizados pelo Ae. aegypti, preferindo, porém os situados no peridomicílio. Corroborando com BALESTRA et al. (2008), os nossos dados demonstraram 53\% de positividade de Ae. albopictus na área urbana e $38 \%$ de ocorrência nos depósitos artificiais (outros = tipo I). Urbinatti (2004) aponta a presença freqüente de Ae. albopictus tanto em áreas de proteção ambiental como em áreas alteradas, reforçando a evidência de sua ampla valência ecológica em colonizar ecótopos naturais e artificiais. Nossos resultados também demonstraram a positividade na área de interface representando uma zona mista entre área rural e urbana.

Silva \& Silva (1999) relataram que o Ae. aegypti é atualmente o mosquito que apresenta maior dispersão em áreas urbanas no mundo. Contudo, a perspectiva de controle desse vetor, na atualidade, esbarra na sua grande capacidade adaptativa a condições adversas, como o desenvolvimento em águas poluídas, e a qüiescência dos ovos em ambientes inóspitos. Pereira (1995) verificou que na cidade de Santos, após extensas campanhas educativas para eliminação de criadouros "convencionais" vem se registrando a predominância de focos de Ae. aegypti em ralos e canaletas de drenagem pluvial, em bairros residenciais com adequada estrutura urbana e em plena estação epidêmica. No Brasil têm-se identificado vasos de plantas, pneus, caixas d' água, floreiras em cemitérios, como criadouros preferenciais das espécies de vetores, com diferentes padrões conforme a época do ano investigada (Chiaravalloti Neto 1997; MACoris et al. 1997). Como já demonstrado na literatura, observou-se neste estudo uma presença urbana do Ae. aegypti com um percentual de 60\% de ocorrência na área urbana e tendo o deposito I como criadouro preferencial em todas as áreas de estudo (urbana, rural e interface).

Quanto à presença de uma espécie em relação à outra a média de 13,45 e 6,9 para Ae. aegypti e Ae. albopictus respectivamente para a área urbana demonstrou a presença efetiva de Ae. aegypti para a área urbanizada. A média de 5,4 e 5,8 para as mesmas espécies, na zona rural foi equivalente, não demonstrando preferência de uma espécie em relação à outra. $\mathrm{Na}$ interface, área de transição, não foi significativa a presença 
de Ae. albopictus (x = 0,3) em relação às outras áreas. Estes dados permitiram concluir que esse mosquito participa da fauna regional, utilizando-se de depósitos diversos decorrentes de diferentes graus de transformação do ambiente. Estes dados demonstraram a necessidade de identificar e possuir um controle mais específico dos depósitos naturais e, eliminar os possíveis depósitos artificiais especiais, passíveis de se tornarem criadouros do mosquito transmissor do dengue.

\section{Agradecimentos}

Os autores agradecem o suporte financeiro da Fundação Educacional Severino Sombra/FUSVE e da Fundação Nacional de Desenvolvimento do Ensino Superior Particular/FUNADESP.

\section{REFERÊNCIAS}

Albuquerque, C.M.R., M.A.V. Melo-Santos, M.A.S. Bezerra, R.M.R. Barbosa, D.F. Silva \& E. Silva, 2000. Primeiro registro de Aedes albopictus em área de Mata Atlântica, Recife, PE, Brasil. Revista de Saúde Pública, 34: 314-315.

Balestra, R.A.M., R.O. Pereira, M.J.S. Ribeiro, J.S. Silva \& J. Alencar, 2008. Ocorrência de Aedes (Stegomyia) albopictus (Skuse) em Área Urbana do Estado do Tocantins. Neotropical Entomology, 37 (2): 233-235.

Becnell, J.J., J. Garcia \& M. Johnson, 1996. Effects of three larvicidas on production of Aedes albopictus based on removal of pupal exuviae. Journal America Mosquito Control Association, 12: 499-502.

Chiaravalloti Neto, F., 1997. Descrição da colonização de Aedes aegypti na região de São José do Rio Preto, SP. Revista Sociedade brasileira Medicina Tropical, 30: 279-285.

Chiavaloti Neto, F., M.R. Dido, A.A.C. Barbosa. \& M. BATTIGAGLIA, 2002. Aedes albopictus na região de São José do Rio Preto, SP. Revista Sociedade Brasileira Medicina Tropical, 35: 351-57.

Clements, A.N., 1992. The Biology of Mosquitoes. Development, Nutrition and Reproduction. Vol. 1, 509 pp. London, Chapman and Hall.

Consoli, R.A.G.B. \& R. Lourenço-de-Oliveira, 1994. Principais mosquitos de importância sanitária no Brasil. Editora FIOCRUZ, Rio de Janeiro, 225p.

Donalisio, M.R. \& C.M. Glasser, 2002. Vigilância Entomológica e Controle de Vetores de Dengue. Revista Brasileira de Entomologia, 5: 260-272.

Ferreira Neto, J.A., M.M. Lima \& M.B. Aragão, 1987. Primeiras observações sobre o Ae. albopictus no Estado do Espírito Santo, Brasil. Caderno de Saúde Pública, Rio de Janeiro.

Focks, D.A., S.R. Sackett, D.L. Bailey \& D.A. Dame, 1981. Observations on container-breeding mosquitoes in New Orleans, Louisiana, with an estimate of the population density of Aedes aegypti (L.). American Journal of Tropical Medicine and Hygiene, 30:1329-1335.

Forattini, O.P. 1962. Entomologia Médica. Vol. 1, Faculdade de Higiene e Saúde Pública, S. Paulo, 662 pp.

Forattini, O.P. 1998. Mosquitos Culicidae como vetores emergentes de infecções. Revista de Saúde Pública, 32: 497502.

Forattini, O.P. 2002. Culicidologia Médica. Identificação, Biologia, epidemiologia. Ed. Universidade de São Paulo, Brasil. Vol.2, 864pp.

Frankie, G.W. \& L. E. Ehler, 1978. Ecology of insects in urban environments. Annual Review of Entomology, 23: 367-387.

Gilotra, S.K., L.E. Rozeboon, \& N.C. Bhattacharya, 1967. Observations on possible competitive displacement between populations of Aedes aegypti Linnaeus and Aedes albopictus Skuse in Calcutta. Bullettin World Health Organ, 37: 437-46.

Gomes A.C., O.P. Forrattini, I. Kakitani, C.C.A. Marques, D. Marucci \& M. Brito, 1992. Microbiotas de Aedes albopictus na região do Vale do Paraíba, Estado de São Paulo, Brasil. Revista Saúde Pública, 26:108-18.

Gomes, A.C. \& G.R.A.M. Marques, 1988. Encontro de criadouro natural de Aedes (Stegomyia) albopictus (Skuse), no Estado de São Paulo, Brasil. Revista Saúde Pública, 22: 245.

Gomes, A.C., M.D. Bitencourt, D. Natal, P.L.S. Pinto, L.F. Mucci, M.B. Paula, P. R. Urbinatti \& J.M.S. Barata. 1999. Aedes albopictus em área rural do Brasil e implicações na transmissão de febre amarela silvestre. Revista Saúde Pública, 33: 95-97.

Gonçalves, K.S. \& M.C. Messias, 2008. Ocorrência de Aedes (Stegomyia) aegypti (Linnaeus, 1762) (Insecta, Diptera, Culicidae) em bromélias, no município do Rio de Janeiro (Rio de Janeiro, Brasil). Biota Neotropica, 8: 235-237.

Hawley, W.A., 1988. The biology of Ae. albopictus. Journal American Mosquito Control Association, 1: 1-40.

Instituto Brasileiro de Geografia e Estatística (IBGE) (29 de agosto de 2008). Página visitada em 23 de setembro de 2008.

Knudsen, A.B. \& R. Slooff, 1992. Vector-borne disease problems in rapid urbanization: new approaches to vector control. Bulletin World Health Organ, 70: 1-6.

Lopes, J., E.A.C. Martins, O. Oliveira, V. Oliveira, B.P. Oliveira Neto \& J.E. Oliveira, 2004. Dispersion of Aedes aegypti (Linnaeus, 1762) and Aedes albopictus (Skuse, 1894) in the rural zone of north Paraná State. Brazilian Archives of Biology and Technology, 47: 739-746.

Lopes, J., M.A.N. Silva, A.M. Borsato, V.D.R.B. Oliveira \& F.J.A. Oliveira, 1993. Aedes (Stegomyia) aegypti L. e a culicídeos fauna associada em área urbana da região Sul, Brasil. Revista de Saúde Pública, 27: 326-333.

Macoris, M. L. G., C.A.B. Mazine, M.T.M. Andrighetti, Yasumaro, S., M.E. Silva, M.J. Nelson, \& P. J. Winch, 1997. Factors favoring houseplant container infestation with Aedes aegypti larvae in Marilia, SP, Brazil. Revista Panam Salud Publica, 1: 280-6.

Miller, B. R. \& M.E. Ballinger, 1988. Aedes albopictus mosquitoes introduced into Brazil: vector competence for yellow fever and dengue viruses. Transactions of the Royal Society of Tropical Medicine and Hygiene, 82: 476-7.

Mitchell, C.J., 1991. Vector competence of north and South American strains of Aedes albopictus for certain arboviroses: A review. Journal of the American Mosquito Control Association, 7: 446-451.

Natal, D., 2002. Bioecologia do Aedes aegypti. Biológico, São Paulo, 64: 205-207.

O'Meara, G.F., L.F. EVANS JR, A.D. GETTMAN \& J.P., 1995. Spread of Aedes albopictus and decline of Ae. aegypti (Diptera: Culicidae) in Florida. Journal Medical Entomology, 32: 554-62.

Pereira, M. \& G.L. Barbosa, 1995. Pesquisa larvária e positividade de recipientes com Aedes aegypti e Aedes albopictus no Estado de São Paulo. XXXI Congresso da Sociedade Brasileira de Medicina Tropical, São Paulo.

Silva, H.H.G. \& I.G. Silva, 1999. Influência do período de qüiescência dos ovos sobre o ciclo de vida de Aedes aegypti (Linnaeus, 1762) (Diptera, Culicidae) em condições de laboratório. Revista Sociedade Brasileira Medicina Tropical, 32: 349-355.

Souza-Santos, R., 1999. Fatores associados à ocorrência de formas imaturas de Aedes aegypti na Ilha do Governador, Rio de Janeiro, Brasil. Revista da Sociedade Brasileira de Medicina Tropical, 32: 373-382.

Taveira, L.A., L.R. Fontes\& D. Natal, 2001. Manual de diretrizes e procedimentos no controle do Aedes aegypti. Prefeitura Municipal de Ribeirão Preto, 6op.

Tun-Lin, W., B.H. Kay \& A. Barnes, 1995. Understanding productivity, a key to Aedes aegypti surveillance. American Journal of Tropical Medicine and Hygiene, 53: 595-601.

Urbinatti, P.R., 2004. Observação ecológica do Aedes albopictus em área de proteção ambiental e urbana da periferia na grande São Paulo. Tese de Doutorado. 
Faculdade de Saúde Pública da Universidade de São Paulo. http://www.teses.usp.br/teses/disponiveis/6/6132/tde-04032005-091946

Varejão, B.M, B.S. Santos, R.H. Rezende, C.L. Bevilacqua \& A. Falqueto, 2005. Criadouros de Aedes (Stegomya) aegypti (Linnaeus, 1762) em bromélias nativas na cidade de Vitória, Recebido em: 05/06/2008 ES. Revista da Sociedade Brasileira de Medicina Tropical, 38: 238-240.

\section{Como citar este artigo:}

Soares, V.A.R.C., W.C. Rodrigues \& M.M.O. Cabral, 2008 Estudo de áreas e depósitos preferenciais de Aedes albopictus (Skuse, 1894) e Aedes aegypti (Linnaeus, 1762) no Município de Paracambi - Rio de Janeiro, Brasil. EntomoBrasilis, 1(3): 63-68. www.periodico.ebras.bio.br/ojs

Aceito em: 14/11/2008

$* * * * * * * * * * * * *$ 